\title{
Confianza epistémica y conflicto epistémico
}

\author{
LINDA ZAGZEBSKI \\ Departamento de Filosofía \\ Universidad de Oklahoma \\ lzagzebski@ou.edu
}

\begin{abstract}
Resumen: En este trabajo se argumenta que el deseo natural de alcanzar la verdad hace que la confianza epistémica en uno mismo sea una exigencia racional para todos, y que existen fundamentos para confiar en muchas de mis disposiciones emocionales que son paralelos a los fundamentos que tengo para confiar en mis facultades epistémicas. Entonces, tanto la confianza epistémica en uno mismo, como la confianza en mi emoción de admiración, me comprometen a confiar en otros. Parece que uno está comprometido con un principio de la confianza, de acuerdo con el cual cualquier razón que tengo para confiar epistémicamente en mí mismo es una razón que se aplica a muchas otras personas, y no tengo una razón para pensar que soy epistémicamente privilegiado. Pero, entonces, ¿cómo debo manejar los conflictos entre mis propias creencias y las creencias de personas en quienes confío? Puesto que el conflicto surge de confiar en el principio de la confianza, es racional resolver el conflicto en favor de aquello en lo que más confío después de reflexionarlo.
\end{abstract}

Palabras clave: confianza en uno mismo, confianza en emociones, confianza en otros, compromiso racional

\begin{abstract}
In this paper I argue that the natural desire for truth makes epistemic self-trust a rational requirement, and there are grounds for trust in many of my emotional dispositions that parallel the grounds for trust in my epistemic faculties. I then argue that both epistemic self-trust and trust in my emotion of admiration commit me to trusting others. It appears that I am committed to a principle of trust according to which any reason I have for epistemic trust in myself is a reason that applies to many other people and I have no reason to think of myself as epistemically privileged. How then should I handle conflict between my own beliefs and the beliefs of persons I trust? I argue that since the conflict is generated from trust in the principle of trust, it is rational to resolve conflict in favor of what I trust the most upon reflection.
\end{abstract}

Key words: self-trust, trust in emotions, trust in others, rational commitment

\section{La confianza en uno mismo}

Deseo comenzar con una verdad obvia: existe un deseo natural de alcanzar la verdad. Al hablar de él me refiero simplemente al deseo de descubrir cómo es el mundo, y cuando digo que es un deseo natural 
quiero decir que virtualmente todos lo tienen. ${ }^{1}$ De modo que virtualmente todos tienen el deseo de descubrir cómo es el mundo y pienso también que virtualmente todos piensan que ese deseo puede satisfacerse. Así pues, además del deseo natural de alcanzar la verdad hay una creencia natural de que ese deseo natural puede satisfacerse.

Virtualmente todos tienen esa creencia, incluyendo el escéptico. El escéptico radical argumenta que esa creencia no está apoyada en la razón ni en otras facultades naturales, pero rara vez, si es que alguna vez ocurre, alguien abandona esa creencia. Tal vez los pirrónicos tuvieron éxito en abandonarla, pero incluso ellos reconocieron que el escepticismo no es un estado natural, pues tenían que llevar a cabo una terapia para alcanzarlo.

No obstante, el escéptico está en lo correcto acerca de algo: no cuento con ninguna forma que no sea circular para determinar que mis facultades en su conjunto me entregan la verdad. Pero tendría que estar muy confundida para esperar tal cosa. El hecho de que no haya una manera no circular de decir que mis facultades son en conjunto fiables se sigue de la suposición de que la fiabilidad en cuestión es una relación entre nuestras facultades tomadas en conjunto y algo que, al menos en principio, es independiente de los productos de esas facultades. Es usual llamar a ese algo "el mundo fuera de nuestras mentes", pero la cuestión no depende de que el mundo sea externo.

Cuento con una forma circular de determinar que mis facultades son fiables. Apoyándome en una facultad puedo determinar que otra facultad es condicionalmente fiable (es decir, fiable dada la fiabilidad de la otra facultad), asimismo puedo decir que la misma facultad es fiable a través del tiempo. Poseo evidencia condicional de mi fiabilidad en algunas ocasiones y evidencia condicional de que no soy fiable en otras ocasiones. Determino la fiabilidad condicional de una facultad confrontando los resultados de esa facultad con los resultados de otras facultades, o de la misma facultad en otras ocasiones. Verifico una percepción apoyándome en otra percepción, mis creencias previas y el testimonio de otros; verifico un recuerdo apoyándome en la percepción, otros recuerdos, creencias y testimonio; verifico el testimonio apoyándome en la percepción, la memoria y creencias previas, y así sucesivamente.

Doy por sentado que una creencia es el resultado de un conjunto de facultades; es el resultado de al menos una y con frecuencia de más

${ }^{1}$ Pienso, además, que hay algunas implicaciones normativas del hecho de que sea un deseo natural: es bueno, o por lo menos no es malo, no es algo a lo que debamos resistirnos. Pero el tema de la normatividad de lo natural no figura en este trabajo. 
de una facultad. Verificamos nuestras creencias de la misma manera en que verificamos los resultados de nuestras facultades sensoriales y de nuestra memoria: las verificamos confrontándolas con los resultados de otras facultades, o de la misma facultad en otras ocasiones. Usualmente verifico una creencia confrontándola con otras creencias mías, conjuntamente con el uso de mis facultades. Al hacer esto siempre tengo que presuponer la fiabilidad de al menos una facultad que confirma alguna de mis creencias. Esto es verdadero aun cuando alguna versión fuerte de fundacionismo sea correcta. Tal vez existan algunas creencias que no necesiten ser verificadas apoyándonos en otras creencias. Aunque fuese así, necesito confiar en la facultad que produce las creencias fundacionales para poder confiar en esas creencias. Mi razón para pensar que el resultado de cualquiera de mis facultades es verídico es condicional, y mi razón para pensar que las facultades que producen esos resultados son fiables es condicional.

Una vez que veo que no hay un apoyo que no sea circular para la creencia natural de que el deseo natural de verdad puede satisfacerse, puedo retener esa creencia sólo si tengo confianza en mí misma, confianza en aquellas de mis facultades que pienso que me conducen a la verdad. A lo que me refiero al hablar de confianza en uno mismo es un estado en el que tengo la misma confianza que tendría si tuviese un apoyo que no fuera circular para la creencia de que mis facultades son en conjunto fiables. No tengo un apoyo tal, pero actúo como si lo tuviera, y tengo la misma actitud hacia mis facultades y sus productos que la que tendría si lo tuviera. La confianza epistémica en uno mismo es una actitud optimista; conduzco mi vida como si tuviera una razón no circular para pensar que soy fiable.

Generalmente la confianza ocurre cuando nos falta algo que en otras condiciones querríamos tener; por ejemplo, una prueba o un tipo fuerte de justificación. Pero una de las características interesantes de la confianza es que nos ofrece ventajas que no tendríamos si contáramos con una prueba. La confianza no es simplemente una actitud de segunda categoría a la cual nos replegamos cuando no tenemos lo que realmente queremos. La confianza crea y fortalece vínculos entre las personas, y la confianza en uno mismo nos vincula con nosotros mismos.

Pienso que la confianza epistémica en uno mismo tiene dos partes: (a) doy por supuesta la fiabilidad general de mis facultades, y (b) de hecho confío en mis facultades. Nótese que (a) no es suficiente para (b): puedo pensar que alguien o algo es fiable sin confiar en eso o en esa persona. Esto puede suceder si hay algo más que también creo que es fiable y puedo apoyarme en ello en lugar de apoyarme en lo otro, 
también puede suceder si no necesito confiar en nada para formar una creencia, porque puedo omitir tener esa creencia. Pero suponiendo que no puedo omitir tener la creencia natural de que mi deseo natural de alcanzar la verdad puede satisfacerse, y dado que no hay nada en lo que pueda confiar que no sean mis facultades tomadas en conjunto, se sigue que necesito confiar en mis facultades tomadas en conjunto. Dado (a), cuento con una presunción en favor de la veracidad de los resultados de mis facultades, hasta que se muestre lo contrario mediante un uso ulterior de mis facultades. Dado (b), mi actitud es confiar en mis facultades al creer, deliberar y actuar; en pocas palabras, al llevar una vida humana ordinaria.

Aquí no estoy hablando de todos los tipos de confianza en uno mismo, sino de la confianza epistémica en uno mismo, y ni siquiera de toda ésta. Me concentro en la confianza con respecto a la conexión entre, por un lado, mis facultades y los productos de éstas y, por el otro, la verdad. Más adelante haré notar algunas maneras en las que la confianza epistémica en uno mismo, en este sentido, necesita extenderse para incluir la confianza en facultades o poderes que generalmente no se clasifican como epistémicos; pero el tipo central de confianza del que estoy hablando es la confianza en la fiabilidad de facultades epistémicas. Trato mis poderes y los datos sobre los que ejercito esos poderes como si me condujeran a satisfacer mi deseo natural por la verdad.

La confianza en uno mismo requiere que haya ciertas cosas en las que tengo que confiar antes de que cuente con evidencia. Una de las cosas en las que tengo que confiar antes de tener evidencia es que la evidencia conduce a la verdad. Tengo evidencia de que la evidencia conduce a la verdad, pero esa evidencia también es condicional: necesito confiar en que existe la conexión entre la evidencia y la verdad para obtener evidencia de que la evidencia conduce a la verdad. Cuando llego a saber que la evidencia $E$ indica la verdad de $p$, lo que de hecho llego a saber es que la evidencia $E$ conduce a más evidencia en favor de $p$, suficiente para que pueda declarar que $p$ es verdadera. (Esto no significa que cuanta más evidencia tenga en favor de $p$, tanto más probable es que $p$ sea verdadera.) La conexión evidencia-verdad es algo en lo que debo confiar. Alguien podría pensar que solamente deberíamos confiar en la evidencia y que solamente deberíamos creer cuando tenemos suficiente evidencia. Tal persona confiaría en el evidencialismo. No sé si alguien haga eso, ni siquiera si alguien pueda hacerlo.

La evidencia no es lo más profundo en lo que confío epistémicamente. Ya he dicho que tengo que confiar en mis facultades epistémicas; también confío en otras cosas relativas a mí misma antes de tener evi- 
dencia de que son conducentes a la verdad. En general confío en lo que llamo meticulosidad. Uso la palabra "meticulosidad" [conscientiousness] para referirme a cierta actitud de amor a la verdad y de tratar de conseguirla; asumo que esa actitud incluye, entre otras cosas, ser cuidadoso, concienzudo, de mente abierta, justo con quienes tienen una opinión contraria. Para mis propósitos no importa cuáles sean las cualidades y comportamientos cognoscitivos específicos que son meticulosos, lo que importa es que confíe en cierta actitud de la mente y que confíe en los comportamientos y hábitos mentales a los cuales esa actitud conduce. Llamo a esto "meticulosidad". (La palabra 'meticulosidad' podría sugerir a algunas personas la idea del deber, pero pienso en ella como una forma de preocuparse por la verdad y de tratar de obtenerla, no como una forma de deber.) Confiar en la meticulosidad es confiar en que existe una conexión entre tratar de obtener la verdad y tener éxito. Supongo que tengo evidencia de que ser meticuloso es conducente a la verdad, pero confío en la meticulosidad antes de tener evidencia porque confío en ella para obtener evidencia; confío más en evidencia adquirida de manera meticulosa que en evidencia adquirida sin meticulosidad. Pienso, además, que confiar en la meticulosidad es más básico que confiar en la evidencia, porque confío en la evidencia en virtud del hecho de que confío en la meticulosidad, y no viceversa. Pero mi tesis principal en este punto es que no tengo una razón que no sea circular para confiar ni en la conexión evidencia-verdad ni en la conexión meticulosidadverdad. Tengo evidencia de que la evidencia conduce a la verdad, y cuando soy meticuloso tengo razón para pensar que la meticulosidad conduce a la verdad, pero no puedo escapar al hecho de que confío en que lo que estoy haciendo cuando soy meticuloso conduce a la verdad.

Hay otras cosas en las que confío epistémicamente antes de tener evidencia de que son conducentes a la verdad. Confío en mis emociones. Tal como las entiendo, las emociones pueden relacionarse de manera apropiada o inapropiada con sus objetos intencionales. Hay cosas que es apropiado temer (para ciertas personas en ciertas circunstancias), y hay cosas que es inapropiado temer. Hay objetos propios de la piedad, el enojo, el amor, el desprecio, la simpatía, la indignación, el remordimiento, y hay objetos inapropiados de estas emociones. Por lo menos a grandes rasgos esto es verdad. (Tal vez hay algunas emociones que siempre son inapropiadas; por ejemplo, la envidia o el odio.) Confiar en mis emociones es más complicado que confiar en mis facultades sensoriales y en mi memoria, esto se debe a que hay emociones tanto entre las cosas en las que más confío como entre las cosas en que menos confío. 
Muchas creencias se fundan en emociones. Probablemente algunos juicios morales están en esta categoría; confiamos en ellos aun cuando no podamos decir por qué confiamos en ellos. Lo más que podemos decir es que tenemos cierta emoción; tal vez repulsión, o desagrado o incluso horror, y que confiamos en esa emoción. Así, formamos un juicio que se basa, al menos en parte, en confiar en una emoción. El psicólogo Jonathan Haidt, quien ha intentado descubrir si la gente puede dar siempre razones a favor de sus juicios morales, diseñó algunos ejemplos. En uno de los casos usados en sus estudios se les dice a los sujetos que imaginen que un automóvil mata al perro de una familia frente a su casa; alguien dice a los miembros de la familia que la carne de perro es comestible, así que destazan el cuerpo del perro, lo cocinan y se lo cenan. La mayoría de la gente en los estudios de Haidt declara que ese acto es incorrecto, pero les resulta difícil explicar por qué lo es. Se les pueden ocurrir razones, pero en general las razones se vienen abajo y finalmente la gente se da por vencida y declara que está segura de que el acto es incorrecto antes de encontrar razones para justificar su juicio. Tienen una reacción visceral; algo así como la repugnancia o el asco. ${ }^{2}$ Según interpreto los resultados de estos estudios, la gente confía en su juicio aunque no tenga un razonamiento para apoyarlo, y su confianza en sus reacciones viscerales es lo suficientemente fuerte para apoyar su confianza en sus juicios morales. Dudo que tengan evidencia de que su reacción visceral sea una facultad fiable, pero, aun cuando tengan esa evidencia, su confianza no se basa en ella. Desde luego, sea lo que fuere que haga repugnante comerse al perro que se tiene por mascota, está relacionado con experiencias pasadas e imitación de las reacciones emocionales de otros; no obstante, es parte de la confianza en uno mismo. Por supuesto, este ejemplo no muestra que en este caso debamos confiar en la emoción, pero sí revela que la mayoría de la gente de hecho confía en ella.

Es obvio que en muchas ocasiones tenemos razones para no confiar en las emociones. Tenemos evidencia, suministrada por la investigación sobre el cerebro, de que las rutas neuronales que conducen a la experiencia de algunas emociones básicas, como el miedo, operan rápidamente en paralelo con las rutas más lentas que conducen a la corteza cerebral. Esto explica por qué podemos experimentar una emoción antes de que hayamos tenido tiempo de evaluar íntegramente tanto la

${ }^{2}$ Haidt investiga las respuestas de la gente a los tabúes y menciona la investigación a la que aquí aludo en su libro The Happiness Hypothesis: Finding Modern Truth in Ancient Wisdom (Basic Books, Nueva York, 2006, p. 42). En el capítulo 9 del mismo libro regresa a la explicación de las respuestas emocionales a los tabúes. 
situación que da lugar a la emoción como si la respuesta emocional es apropiada. Por mucho, nuestras emociones tienden a ser respuestas exageradas a las situaciones; son exageradas tanto en su rango de generalidad (e.g. nos dan miedo las cosas temibles, pero también nos dan miedo muchas cosas que no son temibles), como en su intensidad; así que mucha gente no confía en ellas. Pero sería un error hacer esa inferencia. Una respuesta exagerada puede ser una exageración de la respuesta correcta. De hecho, en general no decimos que algo sea exagerado a menos que sea demasiado de aquello que es lo correcto. Llegamos a sentir demasiada aversión, demasiado enojo, demasiado miedo, a estar demasiado cautivados cuando nos enamoramos, y así sucesivamente, pero de eso no se sigue que las emociones sean en general inapropiadas. Si interpretamos las emociones como parte de una red de facultades que incluye la evaluación cognoscitiva del estímulo emocional, así como el sentimiento inmediato generado por rutas neuronales alternas, entonces creo que no tenemos razón para pensar que en general las emociones no son fiables.

De hecho, necesitamos confiar en las emociones al menos por dos razones. Los ejemplos en los estudios psicológicos de Haidt nos dan la primera razón: muchas creencias se fundan en emociones, específicamente creencias morales y otras creencias que llevan a la acción. El miedo a una situación funda la creencia de que debo escapar, la compasión por alguien funda la creencia de que debemos ayudarle, la aversión hacia un acto o una situación me lleva a evitarla, y así sucesivamente. La confianza en uno mismo que necesitamos para actuar a veces requiere confianza en creencias que a su vez dependen de que confiemos en emociones. Si la confianza epistémica en uno mismo incluye la confianza en las creencias en las que se funda la acción, entonces la confianza epistémica en uno mismo requiere confiar en las emociones de uno mismo.

En segundo lugar, nuestros fundamentos para confiar en las emociones son los mismos que tenemos para confiar en facultades como la percepción, la memoria y la razón: no hay fundamentos que no sean circulares para creer que son fiables, pero hay evidencia (circular) de que nuestras disposiciones emocionales que sobreviven a la reflexión son fiables, del mismo modo que hay evidencia circular de que nuestras facultades de percepción y memoria son fiables. La evidencia no es tan clara y convincente como la evidencia en favor de la fiabilidad de ciertos tipos de percepción, memoria y razonamiento, pero hay muchos casos en los que tenemos evidencia de la misma clase y de la misma fuerza en favor de la fiabilidad de ciertas emociones, en cierto espectro 
de circunstancias, que la que tenemos en favor de la percepción y la memoria. Confiamos en lo que pensamos que vemos cuando miramos con cuidado en buenas condiciones ambientales, y si otros están de acuerdo con nosotros, tomamos esto como una confirmación. De manera similar, confiamos en lo que sentimos cuando sentimos admiración o compasión o desprecio, y tomamos el acuerdo con otros como una confirmación. En ambos casos tiene que haber acotaciones, y sin duda hay muchos más casos en los que no confiamos en una emoción que en los que no confiamos en una percepción sensorial; pero tenemos la misma clase de fundamentos para confiar en una emoción que los que tenemos para confiar en una percepción o en un recuerdo. No podemos comprobar su fiabilidad de una manera que no sea circular, pero a veces su fiabilidad puede ponerse a prueba de una manera circular y pasar la prueba.

\section{La confianza en otros}

A continuación deseo argumentar que las bases que he mencionado que tenemos para confiar en uno mismo también son bases para confiar en otros, aunque no todas las diferentes maneras que tengo de confiar en mí misma me conducen a confiar en otros de igual manera. Antes distinguí dos aspectos de la confianza en uno mismo: pensar que uno es fiable y de hecho confiar en uno mismo. Para llevar una vida normal debo hacer ambas cosas, pero es posible no hacer ninguna de las dos con respecto a otros. El egoísmo epistémico es posible en algún grado. Ahora quiero argumentar que (1) estoy comprometida a pensar que los otros son epistémicamente dignos de confianza (fiables), y (2) que de hecho estoy comprometida a confiar en ellos (apoyarme en ellos) epistémicamente. De modo que confiar en mí misma me compromete a confiar en otros.

\section{(A) Argumento en favor de (1)}

(i) He argumentado que debo pensar que en conjunto mis facultades son fiables, a pesar de que no tenga una razón que no sea circular para pensarlo. Pero si no tengo razones que no sean circulares para pensar que en conjunto soy fiable, tampoco tengo razones que no sean circulares para pensar que en conjunto soy más fiable que otras personas. Supongo que en un sentido débil de "razón" tengo una razón para confiar en mis facultades en su conjunto. Tal vez simplemente ser un yo o un ser humano es una razón. Pero sé que hay otros yos y otros seres humanos también, y si soy fiable en general sólo porque soy un yo, ellos también lo son y por la misma razón. Tengo que pensar que son fiables 
en general antes de que tenga evidencia para creerlo, del mismo modo en que pienso que yo misma soy fiable antes de que tenga evidencia para creerlo. A esto me compromete el principio según el cual debo tratar casos similares de modo similar.

(ii) Usando mis propias facultades obtengo evidencia, evidencia condicional, de que soy fiable en algunos aspectos y de que no lo soy en otros. Apoyándome en mis propias facultades consigo determinar que a veces y en algunos aspectos soy fiable, y que en otros no lo soy. De la misma manera, tengo evidencia de que algunas personas son más fiables que yo en algunos aspectos y que algunas lo son menos. En la medida en que reconozco que yo misma soy fiable en algunos aspectos porque adquiero evidencia de mi fiabilidad, así también tengo que reconocer que otras personas son fiables en algunos aspectos.

(iii) También argumenté que confío en mí misma antes de tener evidencia de mi fiabilidad; en particular confío en mí misma cuando soy meticulosa — cuando soy cuidadosa y me esfuerzo por obtener la verdad-. Cuando soy meticulosa obtengo evidencia de que soy fiable, pero confío en mí misma antes de obtener esa evidencia. De manera similar, cuando creo algo meticulosamente, descubro que otros son meticulosos. Ellos tienen el mismo tipo de meticulosidad en la que confío en mi propio caso. Cuando actúo meticulosamente llego a creer que otras personas son meticulosas; de modo que si confío en mí misma cuando soy meticulosa y porque soy meticulosa, por esa razón debo considerar que otros, que también son meticulosos, son fiables. Si la cualidad en la que confío en mi propio caso no es la meticulosidad sino alguna otra, se sigue aun la misma conclusión, pues cuando creo algo por tener la cualidad en la que confío, inevitablemente creeré que hay otros que creen de la misma manera.

Nótese que, según mi argumento en los incisos (ii) y (iii), confiar en uno mismo requiere que uno vea que otros son fiables. Puesto que confío en mi propia habilidad para evaluar evidencia, debo confiar en mi evidencia de que otras personas son fiables, y puesto que confío en mí misma cuando soy meticulosa, y por la razón de que soy meticulosa, debo confiar en otros cuya meticulosidad descubro cuando soy meticulosa. La confianza general en uno mismo de la que se habla en (i) conduce de otra manera a creer que otros son meticulosos. Estoy comprometida a tratar casos similares de manera similar y, dado que no hay diferencia relevante entre otros y yo misma, tengo que considerar que en general son fiables si considero que en general yo misma soy fiable. 
De los fundamentos de la confianza que hemos considerado hasta ahora parece seguirse que cualquier razón que tengo para considerar que yo misma soy fiable es una razón que se aplica a mucha otra gente, y mi razón para considerar que yo misma en general soy fiable se aplica a todas las otras personas.

\section{(B) Argumento en favor de (2)}

Alguien podría estar de acuerdo en que las rutas que nos llevan de la confianza en uno mismo a la confianza en otros tienen éxito en establecer (1): que estoy comprometida a pensar que los otros son fiables. Pero de ello no se sigue que esté comprometida a de hecho confiar en ellos. No tengo ninguna obligación de confiar en todos aquellos a quienes considero fiables. Si prefiero confiar en mí misma y no en los otros, o confiar en mí misma más que en los otros, ¿por qué no he de hacerlo? ¿Puedo rehusarme razonablemente a confiar epistémicamente en los demás?

Pienso que no puedo hacer tal cosa si me preocupa la verdad. Si no confío en los demás, tengo que ignorar mi evidencia de que otras personas son fiables, y tengo que ignorar las creencias a las que llego cuando soy meticulosa - cuando formo creencias a partir de una preocupación por alcanzar la verdad-. La única manera de hacer eso es dándole importancia a mis facultades y a sus productos más que a la verdad; darle importancia a mi evidencia, no porque conduzca a la verdad, sino porque es mi evidencia; darle importancia a mis creencias formadas de manera meticulosa, no porque sea meticulosa (porque me preocupe la verdad), sino porque son mis creencias. Debo considerar que yo misma soy fiable, no porque sea un yo [self], sino porque se trata de mí misma [myself], y eso es muy implaudible. No se trata de un mero egoísmo epistémico, es un egoísmo ético en el ámbito del intelecto. Ese problema surge siempre que confío en mí misma más que en otros, cuando mi evidencia o el uso meticuloso de mis facultades indica que ellos son tan fiables o meticulosos como yo. En la medida en que confío en mí misma más de lo que confío en ellos, mi razón no es epistémica. De modo que valoro mis facultades más que la verdad. Eso es egoísmo ético.

Asumiendo que no quiero ser una egoísta ética, estoy racionalmente comprometida a confiar en los demás sobre las mismas bases sobre las que confío en mí misma. Así pues, parece que dadas nuestras consideraciones hasta el momento la razón que tengo para confiar en mí misma en general se aplica a todas las demás personas. Debo tener una actitud revisable de confianza general en todas las demás personas si 
tengo esa actitud hacia mí misma, y tengo esa actitud hacia mí misma. Es más, mis razones para confiar en mí misma en muchas ocasiones particulares se aplican a muchas otras personas. Por lo tanto, parece que nos vemos conducidos a aceptar alguna versión del principio que de manera tentativa formularé como sigue:

Principio de la confianza: cualquier razón que tengo para confiar epistémicamente en mí mismo es una razón que se aplica a muchas otras personas, y la razón que tengo para confiar en general en mí mismo se aplica a todas las otras personas. No tengo ninguna razón para pensar que soy epistémicamente privilegiado, y no debo tratarme a mí mismo como si fuera epistémicamente privilegiado.

¿Pero no es acaso absurdo el principio de la confianza? ¿Qué pasa con la autoridad de la perspectiva de la primera persona? Sería extraño si ocurriera que estuviese comprometida a pensarme a mí misma como nada más que un repositorio de una porción de evidencia entre muchos otros repositorios de evidencia que poseen otros y que me es accesible; y sería muy extraño si tuviera que pensar que mis facultades no son más que un lote entre muchos otros lotes de facultades en los que debo confiar. Desde luego, todos estarán de acuerdo en que soy especial de algún modo: el uso de mis propias facultades es primario; en cuanto al orden, mis facultades vienen en primer lugar, confío en ellas en primer lugar, y confío en ellas a lo largo de todo el proceso que me lleva a confiar en otros. Pero eso no da a mis facultades ninguna autoridad o privilegio especial, no hace que sean más fiables que otras. También es verdad que en último término soy yo quien decide en qué confiar y en qué no confiar; ¿pero qué con eso? Si para confiar consistentemente en mí misma tengo que confiar en otros, mi autoridad no es más que la autoridad de reconocer que mi propia autoridad resulta socavable. Tengo el privilegio epistémico de reconocer que no tengo ningún privilegio.

En este momento parecería que nos encontramos atrapados en un dilema. Si rechazamos el principio de la confianza, nos vemos conducidos a un egoísmo ético vulgar; pero si lo aceptamos, ello parece hacer que el yo, en cualquier sentido interesante de la palabra, desaparezca. No soy más fiable que la siguiente persona, pero seguro que tengo el derecho de tratarme a mí misma como si fuera epistémicamente privilegiada en algún sentido, aunque sea demasiado fuerte decir que tengo una autoridad epistémica especial. Sin duda que tengo algún tipo de privilegio epistémico con respecto a mí misma. 
No puedo decir mucho más sobre autoridad o privilegio epistémico, así que quisiera regresar a examinar más de cerca la confianza en uno mismo, pues pienso que aún no hemos revisado todas las formas en las que tenemos confianza epistémica en nosotros mismos y la importancia de éstas para confiar en otros. Hay un fundamento más de la confianza en uno mismo que aún no he discutido en lo que toca a sus implicaciones para confiar en otros; me refiero a la confianza en las emociones.

\section{La confianza en las emociones}

He argumentado que confiamos en muchas de nuestras emociones como fundamentos de nuestras creencias y que, puesto que confiamos epistémicamente en nosotros mismos, estamos comprometidos a confiar epistémicamente en otros. Sin embargo, la mayoría de nosotros confía en sus propias emociones más que en las emociones de otros, y no está claro por qué lo hacemos y si es defendible que lo hagamos. Si siento disgusto o repulsión o admiración o compasión o desprecio, tiendo a confiar en esas emociones más que en las emociones similares de otros. Pero no hago esto de manera consistente, pues con frecuencia comparo mis emociones con las de otros con el fin de ayudarme a determinar si son apropiadas. Concedamos que probablemente estamos menos seguros del cognoscitivismo en relación con las emociones que del cognoscitivismo en relación con las creencias, y así es probable que estemos dispuestos a aceptar una mayor variación en las emociones que en las creencias antes de sentir que hay un conflicto. No obstante, soy lo suficientemente cognoscitivista acerca de las emociones para pensar que cuando siento admiración por alguna persona en algún aspecto, si alguien más en cuyas disposiciones emocionales yo confío siente desprecio por esa persona en ese mismo aspecto, surja la molesta sensación de que alguno de los dos está cometiendo un error, y mi seguridad con respecto a mi emoción disminuye. No quiero insistir en que las emociones entran en conflicto justo de la misma manera en que las creencias entran en conflicto, pero el hecho es que las emociones entran en conflicto, y con frecuencia podemos reconocer cuando esto ocurre.

Confiar en las emociones lleva a confiar en otros de una manera diferente que confiar en la evidencia y en la meticulosidad, esto se debe a que, normalmente, no son mis propias emociones lo que me lleva a confiar en las emociones de otra persona. Dije antes que confiar en mi propia evidencia requiere que confíe en mi evidencia de que otros tienen evidencia. Confiar en mis propias creencias formadas de manera 
meticulosa requiere que confíe en las creencias de otros, las cuales creo, al conducirme meticulosamente, que fueron formadas de una manera meticulosa. Pero confiar en mi emoción de desprecio no requiere que confíe de la misma manera en la emoción de desprecio de alguien más. Lo que me conduce a confiar en las emociones de otros es el principio de que debo tratar casos similares de manera similar. Me inclino a pensar que debo adoptar una confianza general revisable con respecto a las emociones de otros, de igual manera como la adopto con respecto a las mías, así como adopto una confianza general revisable con respecto a sus facultades sensoriales, similar a la que adopto con respecto a las mías. Pero esta idea es demasiado cruda y tal vez mi confianza revisable pueda ser de hecho revisada de un modo más bien fácil, aunque no estoy segura de que eso sea razonable. En cualquier caso, lo que estoy sugiriendo es que debo tratar el hecho de que alguien más tiene determinada reacción emocional ante una situación dada como una razón revisable para que yo misma tenga esa reacción, y, si tengo una emoción que entra en conflicto con aquélla, la emoción de la otra persona contará en contra de que mi propia emoción sea apropiada.

Así pues, tenemos dos tipos de razones diferentes para confiar en otros en virtud de que confiamos en cosas acerca de nosotros mismos. Debemos tener una confianza general revisable en las emociones y facultades de otros por el principio de que debemos tratar casos similares de manera similar. Tenemos un argumento más directo a favor de que la confianza en el contenido de nuestra evidencia y en nuestras creencias formadas de manera meticulosa requiere racionalmente que nosotros confiemos en las creencias de otros. Los argumentos en esta última categoría no dependen del principio de que debemos tratar casos similares de manera similar, y por esta razón pienso que son más fuertes que los argumentos en la otra categoría. Sin embargo, hay una emoción tal que confiar en ella me conduce a confiar en las creencias de otros, y que no depende del principio de que debemos tratar casos similares de manera similar. Estoy pensando en un estado emocional que me compromete a confiar en las creencias de los demás directamente, me refiero a la emoción de admiración epistémica. ${ }^{3}$

\footnotetext{
${ }^{3}$ Pienso que esta emoción es un fundamento de la confianza epistémica en uno mismo más profundo que ser meticuloso o que la evidencia. Confío en mi evidencia porque confiar en mi evidencia es lo que hago cuando soy meticulosa y confío en mí misma cuando soy meticulosa. Confío en mí misma cuando soy meticulosa porque admiro epistémicamente la meticulosidad y confío en mi emoción de admiración.
} 
En mi teoría de las emociones, la estructura general de una emoción es la siguiente: La emoción $E$ consiste en sentir de una forma característica con respecto a un objeto que se aprecia como $(E)$-able. La conmiseración es sentir de una manera característica que llamamos conmiseración con respecto a alguien que apreciamos como conmiserable, el miedo es sentir temor con respecto a alguien que apreciamos como temible, el desprecio es sentir desprecio por alguien que apreciamos como despreciable, la reverencia es reverenciar algo que se aprecia como sagrado, la admiración es admirar a alguien que apreciamos como admirable, y así sucesivamente. También pienso que el componente de sentimiento y el componente cognoscitivo de las emociones no pueden separarse en los casos de las emociones que llamo de nivel fundamental, pero eso no es importante para mis propósitos en este trabajo. Todo lo que se necesita es aceptar que en la emoción de admiración hay un sentimiento característico dirigido hacia un objeto que se percibe de cierta manera. Admiro a alguien que veo como admirable, y confiar en mi emoción de admiración es confiar en que la persona que veo como admirable es admirable. Sugiero que lo admirable es algo así como lo imitablemente atractivo. Cuando admiramos a alguien, sentimos una emoción positiva hacia la persona que admiramos; esa emoción conduciría a imitar a esa persona si el resto de las cosas fueran propicias (desde luego, con frecuencia no lo son). De modo que confiar en la emoción de admiración significa tener confianza en que es apropiado sentir el tipo de atracción y deseo de imitar que es intrínseco a la admiración. Confío en mi emoción de admiración antes de tener evidencia de que es fiable. Cuando admiro epistémicamente a otra persona en algún aspecto, siento el deseo de imitar a esa persona en ese aspecto, lo cual puede incluir que forme ciertas creencias.

\section{Conflictos entre la confianza en uno mismo y en otros}

No es usual que notemos aquello en lo que confiamos, ni cuánto confiamos en ello, hasta que algo en lo que confiamos entra en conflicto con alguna otra cosa en la que también confiamos. Confío en mi creencia de que $p$ y en mi evidencia a favor de $p$, pero también confío en mi evidencia de que una persona en quien confío cree que no $p$ y confío en su evidencia a favor de no $p$. Confío en mi creencia meticulosamente formada de que $q$, pero también confío en mi creencia meticulosamente formada de que alguien más cree meticulosamente que no $q$. Confío en mi creencia $r$, pero también confío en mi admiración epistémica y en mi deseo por imitar a alguien que cree no $r$. Confío en mis facultades 
perceptivas y en mis disposiciones emocionales en general, pero también confío en las facultades y disposiciones de otros. Cuando existe un conflicto entre las cosas en las que confío, suele ocurrir que el grado en el que confío en esas cosas cambia de manera inconsciente. Cuando se da un conflicto que persiste, o cuando llega el momento en el que tengo que hacer una elección que requiere que favorezca alguna de las cosas en las que confío sobre otra, entonces me veo forzada a reflexionar sobre la estructura de aquello en lo que confío.

En la medida en que confío en el principio de la confianza, tengo que pensar que no cuento con una razón de principio para resolver a mi favor conflictos entre algo en lo que confío en mí misma y algo en lo que confío en otra persona; pero dando por sentado que confío en la estructura de mi propia confianza, entonces cuento con una razón de principio para resolver el conflicto en favor de aquello en que, después de reflexionar, es en lo que más confío. Esto incluye el grado en el que confío en las cosas particulares en las que confío, y la medida en que una cosa en la que confío depende de algo más en lo que también confío. Desde luego, puede ser que no confíe en el mismo grado en cada una de las partes de la estructura de aquello en lo que confío. Si confío en el principio de la confianza más que en los aspectos de mí misma que generan el conflicto, entonces puede ser razonable resolver el conflicto adoptando una actitud escéptica, pero no hay razón para pensar que ésa sería la respuesta usual. Por ejemplo, supóngase que confío en mi creencia de que en Oklahoma los árboles japoneses de jardín necesitan la sombra de la tarde, pero también confío en que mi amigo cree meticulosamente que los árboles japoneses de jardín prefieren el sol pleno. Adoptaría una posición escéptica hacia mi creencia sobre los requerimientos de sombra de esos árboles sólo si confío en el principio de la confianza más de lo que confío en cualquiera de las creencias que generan el conflicto.

Pero no debo eliminar una creencia a menos que confíe en el principio que llevaría a abandonarla más de lo que confío en la creencia misma. Supóngase, por el contrario, que confío en mi creencia de que ciertos tipos de conducta son equivocados; por ejemplo, el caso que mencioné antes sobre comerse al perro de la casa. Supóngase también que confío en la sabiduría moral de una persona que no responde de manera negativa a tal clase de conducta. Mientras confíe en mi creencia moral o en mi admiración hacia la otra persona más de lo que confío en el principio de la confianza, no tendré ninguna razón para convertirme en una escéptica acerca de la maldad moral de comerse al perro de la casa. Si confío en mi juicio más que en mi admiración hacia el juicio de 
la otra persona, debo retener mi juicio. Si confío más en mi admiración, entonces, ceteris paribus, debería adoptar el juicio de la otra persona. Debo adoptar una actitud escéptica sólo si confío en el principio de la confianza más de lo que confío en cualquiera de los dos aspectos de mi misma que producen el conflicto.

Hasta ahora he considerado los casos más simples de conflicto en el interior de la confianza en uno mismo - confianza en una creencia singular, en una emoción, en un recuerdo o en el principio de la confianza-. Pero muchas de las cosas en las que confiamos se encuentran conectadas en una red que incluye numerosas creencias, emociones, relaciones con otras personas y lealtades a tradiciones que nos dan forma y nos ayudan a interpretar nuestra experiencia. Si una de mis creencias en la red entra en conflicto con algo más en lo que confío, por ejemplo, mi emoción de admiración hacia una persona que tiene una creencia contraria a la mía, entonces no puedo determinar mi respuesta simplemente sopesando cuánto confío en mi creencia y cuánto confío en mi admiración. Confiar en la estructura que tiene la confianza en sí mismo significa que debo considerar cuánto confío en la red de creencias, de emociones y de lealtades en la cual está incrustada la creencia. De modo que la cuestión no es simplemente cuánto confío en la creencia, sino cuánto confío en los otros aspectos de mí misma que tendría que cambiar si cambiara la creencia. Las creencias religiosas se encuentran con frecuencia en esta categoría de creencias que son parte de una red justamente del tipo descrito; pienso que la estructura de la confianza en uno mismo las hace estar mucho mejor atrincheradas que muchas otras clases de creencias, y es razonable que así sea. Así pues, el conflicto entre mi confianza en muchas de mis creencias religiosas y mi confianza en creencias o emociones que me comprometen a confiar en las creencias religiosas de otras personas, no es como el conflicto entre mi confianza en la manera como sumé una columna de cifras y mi confianza en la manera como mi amigo sumó las mismas cifras. Tampoco es como el conflicto entre mi percepción visual y la percepción de otra persona en cuyas percepciones visuales confío, y tampoco es como el conflicto entre mi creencia sobre los méritos de un libro nuevo recientemente publicado y la creencia contraria de un amigo cuyo juicio en la materia admiro. Desde luego, algunas creencias religiosas son así. Es un hecho que sé muy poco acerca de exégesis bíblica. Supóngase que tengo una interpretación muy poco informada de un pasaje del Evangelio y entonces me doy cuenta de que alguien, en cuyo conocimiento experto confío, tiene una interpretación diferente, y no conozco a nadie en quien confíe al menos en la misma medida cuya interpretación 
concuerde con la mía. Si puedo cambiar mi creencia acerca del pasaje sin cambiar ninguna otra cosa en la que confíe más de lo que confío en la otra interpretación, entonces probablemente debería cambiar mi creencia acerca de ese pasaje del Evangelio.

Pero una religión es una práctica compleja, y ello significa que hay muchas creencias, emociones, experiencias y lealtades que se encuentran interconectadas de maneras intrincadas, de modo que cambiar una de ellas comrpomete a la persona a cambiar muchas otras, tal vez incluso la red completa. Y puede ser razonable cambiar la red completa. He aquí dos maneras en las que sería razonable renunciar a una red completa de ese tipo, dado lo que he dicho en este trabajo. Supóngase que admiro a un hindú devoto y que confío en mi admiración por esa persona más de lo que confío en la red de mis creencias, emociones y lealtades cristianas. Dada la estructura de mi confianza en mí misma, sería razonable para mí convertirme al hinduismo, y pienso que para algunas personas la conversión radical es una acción razonable. Pero si estoy en lo cierto acerca de que las creencias religiosas se encuentran con frecuencia profundamente incrustadas dentro de la estructura de la confianza en uno mismo, sería de esperarse que la conversión a esa escala fuera rara. Además, también sería raro que fuera razonable si estoy en lo correcto acerca de que es razonable confiar en la estructura de la confianza en uno mismo.

Existe una manera más en la que el cambio completo de creencias religiosas sería razonable, dada la estructura de la confianza en uno mismo. Supóngase que llego a confiar en el principio de la confianza, tal vez con base en argumentos similares a los que he dado en este trabajo. Si confío en el principio de la confianza más de lo que confío en las creencias o emociones que están en mi red de creencias y emociones religiosas, entonces, la confianza en el principio de la confianza podría llevarme al escepticismo religioso. Supongo que algunas personas renuncian a sus creencias religiosas porque confían en alguna versión del principio de la confianza más de lo que confían en esas creencias y en las otras características de sí mismos que tienen que cambiar cuando renuncian a esas creencias. Pero no veo ninguna razón para pensar que cualquier persona razonable confíe en el principio de la confianza más que en su red de creencias religiosas, ni siquiera que mucha gente razonable lo haga. De hecho, confiar en la estructura de la confianza en uno mismo me lleva a confiar en aquello en lo que confía la gente en quien confío, y no tengo una razón para pensar que en general la gente en quien confío confíe en el principio de la confianza, aunque algunos de ellos lo hagan. Lo que es más probable es que la gente en quien 
confío confíe en el principio de la confianza hasta cierto punto, pero no en su completa generalidad. Tal vez confían en una versión débil del principio; pero, incluso si esto es así, no es probable que confíen en esa versión del principio más que en otras cosas en las que confían. No es difícil que confíen en las creencias religiosas más que en cualquier versión del principio de la confianza que haría razonable abandonar esas creencias.

\section{5. ¿Debo confiar en el principio de la confianza?}

En este trabajo comencé discutiendo la necesidad que tiene una persona razonable de tener confianza en sí misma, dada la creencia natural de que el deseo natural de alcanzar la verdad puede satisfacerse, y he argumentado que la confianza en uno mismo nos compromete a confiar en otras personas de varias maneras diferentes. Hay muchas razones para pensar que aquellos aspectos de uno mismo en los que uno confía: creencias, emociones y actitudes epistémicas como la meticulosidad, me empujan a aceptar el principio de la confianza, el principio según el cual debo confiar en otros tanto como confío en mí misma y por las mismas razones por las que confío en mí misma. Así pues, el principio de la confianza es parte de una red de cosas en las que confío, lo cual me da razón para confiar en él. Confiar en el principio de la confianza me conduce a confiar en la creencias y emociones contrarias de otras personas tanto como confío en las mías, así como en la estructura de aquello en lo que ellos confían tanto como en la estructura de mi propia confianza. Pero parece que hacer esto me conduce en una de dos direcciones, y ninguna de las dos me parece aceptable. Inicialmente, el principio de la confianza me empuja en dirección a intentar encontrar acuerdo con otros, no sólo en lo que respecta a creencias, sino en cualquier otro estado en el que confíe -emociones, principios morales y la estructura de la confianza-. Si confiáramos en el principio de la confianza más que en cualquiera de nuestras creencias, emociones y la estructura de nuestra propia confianza, entonces, en un mundo perfectamente racional, todas nuestras creencias, emociones y principios serían idénticos; y en un mundo de comunicación perfecta, nuestros datos perceptivos también serían idénticos. Una persona se diferenciaría de otra sólo en el hecho de que algunas experiencias son de primera mano para una persona y de segunda mano para todos los demás; pero esto no es suficiente para distinguir a una persona de otra de una manera interesante. Así que me parece que el principio de la confianza nos empuja a intentar convertirnos en la misma persona, y si la confianza 
racional en uno mismo me compromete a confiar en el principio de la confianza, entonces la confianza racional en uno mismo nos empuja a intentar convertirnos en la misma persona. Yo no confío en eso. Ahora bien, desde luego no sucederá que todos nos convirtamos en la misma persona, pues no vivimos en un mundo de comunicación y racionalidad perfectas, pero la pregunta que me parece interesante es si debemos intentarlo. Pienso que la respuesta es no.

Dada la manera como de hecho es el mundo, confiar en el principio de la confianza me da otra opción: el escepticismo. Si confío en el principio de la confianza y descubro que una de mis creencias entra en conflicto con una creencia de alguien en quien confío, y no podemos resolver el desacuerdo, mi confianza en mi creencia se ve socavada, y fácilmente podría ocurrir que sea bastante socavada hasta el punto de que la abandone. Pero el principio de la confianza debe tener el mismo efecto sobre cualquier cosa en mí misma en la que confíe, incluyendo mis emociones y principios morales y epistémicos. En la medida en que confío en el principio de la confianza y me encuentro con gente en quien confío, cuyas emociones, principios morales y epistémicos y grados de confianza en esas partes de ellos mismos entran en conflicto con los míos, y no puedo resolver el conflicto con ellos, debería abandonar esas emociones, principios y grados de confianza. Pero cada vez que abandono una creencia, una emoción o un principio, pierdo una parte de mí misma. La ruta escéptica conduce a un adelgazamiento del yo, y no confío en un principio que tenga esas consecuencias. Es más, si otras personas en quienes confío no confían en el principio de la confianza, eso socava mi motivo para usar ese principio como una razón para abandonar mis creencias y otras partes de mí misma.

Todo lo que puedo hacer es confiar en aquello en lo que confío al conducirme meticulosa y reflexivamente; me parece que hacer esto está muy cerca de lo que Kant quiso decir con ser un agente autónomo. Pero no soy un agente autónomo en otro sentido: dudo que pueda universalizar la estructura de la confianza en uno mismo. Reconozco que diferentes personas terminarán teniendo diferentes creencias y diferentes emociones cuando confíen en la estructura de su propia confianza y sean meticulosas al hacerlo. Pero eso era de esperarse, puesto que no somos todos el mismo yo.

[Traducción de Miguel Ángel Fernández Vargas; revisión de Margarita M. Valdés.]

Recibido el 19 de enero de 2009; aceptado el 23 de marzo de 2009. 